\title{
Desempenho de Estudantes de Medicina em Avaliação de Conhecimento Específico
}

\author{
Performance by Medical Students in \\ Testing Specific Knowledge
}

\section{PALAVRAS CHLIVE:}

- Estudarses de medicina:

- Indisidualidade;

- Conhecimento de resuleados.

KEX-WVRIDS:

- Srudents, Medical;

-Individlualing;

-Knowledge of Results.

Reccbidn cm: 25/07/2004

Ronearvivitulu an:01/10/2003

Roranidectuem: 19/12/2003

Reacaminhach en: 25/06/204

Aprervadis crn:07/07/2004

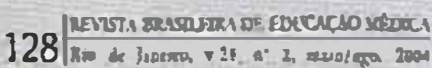

Andy Petroianu'

Bruno Mcllo Rodrigues dos Santos 2 Roberto Martins Gonçalves

\begin{abstract}
RESUMO

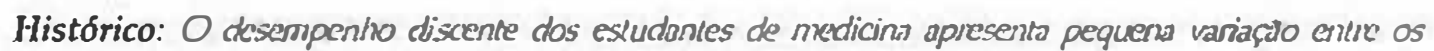
alunos: fintietanio, alguns esiudantes se destacam dos demais, provavilstente devido a caracieristicas individuais. Objetivo: Veljficarse caracteristias pessoajs so importantes no desempenho intekdual de estudantes de medicina. Métodos: Foram estudndos 825 estudantes do $6^{\circ}$ ano da Faculdade de Medicina da UAMIG, caracterizados de acordo com idade, sero, Iateralidade cerebral, cteficiencia visual

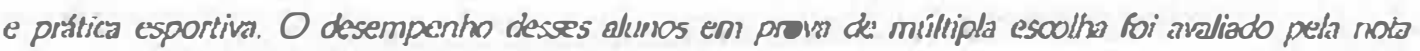
c rempo de realizagero da prova. Resultados: Os estudantes mais jovens e do sexo masculino obtrie ram melhor nota na piova. Os estudantes destios aparentemente terminaram a piova em tempo menor do que os canirolos. Os atunos com defioiencio visual tenderam a abter melthor nota. A prálica de esponk's näo influenciou o desermpentho intulectual. Conclusōes: Os estudanhes de medioinz sta propensos a descmpentio difenenciado crn exame espeoifica de acovrio com calacteristicas individuais.
\end{abstract}

\begin{abstract}
Background: Wertical siudents' performance presents littk. variation from anc student (o) arkilker. Hosvener, sonx students derscristroke greater achieverment, probably dee to perscral characteristics. Objective: To verify whellxes personal characteristics play an important sele in the intellectual porfor. manke of medical sludents. Method: The study mas conducted with 825 students from the siveh year

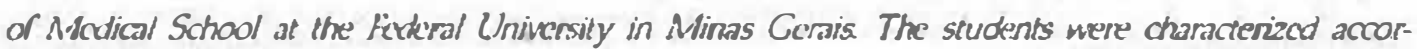
ding to theij agr, gender, cerebral latcuality, visual acuity, and sports actrivity. Thesi pentomance on a

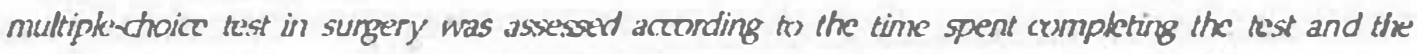
grouk: and was then correvared in individual duaracteristica Results: Shudents under 24 years of age and maks recrived higher goades (m) Itx. re'st. Right-handed students tended to finish the lest in less lirme I/kan kefl-kanders. Siudents requiving cossectrve kense's also got higher grades. Practicing sparts did not infhernce intellectual perfoumance. Conclusion: Medical siudents tend to present different intelkatual pesformance acconting to their individual charocteristics.
\end{abstract}

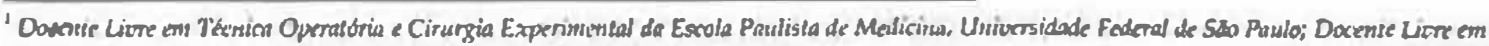

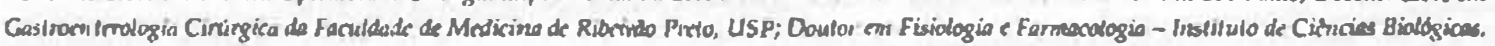

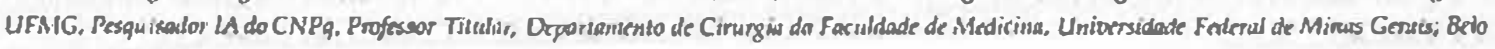
Horizumer, Brissil.

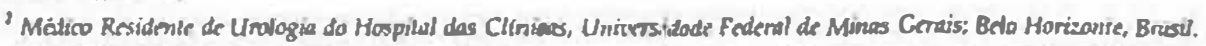

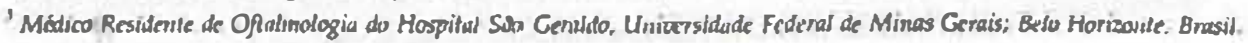




\section{INTRODUÇÃO}

Nos cursos básico e secundário, percebe-se uma grande diferença de desempenho entre os alunos, devido a múltiplos fatores educacionais, intelectuais, sociais e até nutricionais ${ }^{1,2}$. Entretanto, o rigor do concurso vestibular seleciona os alunos com melhor conhecimento, em um nivel de notas que pouco difere entre o primeiro e o último aprovado. Essa equivalência persiste durante todo o curso universitário, em cuja análise se percebe o equilibrio no nivel dos estudantes ${ }^{3,4.5,6}$.

Nesse panorama, sobressaem alguns alunos com desempenhos diferenciados, que tendem para conceitos de avaliação mais elevados. É possivel que atributos peculiares, como interesse específico, talento e treinamento intelectual maior, possam ter papel importante na produtividade discente ${ }^{7,9,9}$. Recorrendo à literatura, encontramos um grande número de artigos que relacionarr. o rendimento estudantil com diferentes fatores, principalmente sociais e de motivaçảo pessoal ${ }^{56,10,34}$. Entretanto, nảo encontramos relaçāo entre a atuação em exames e características individuais dos estudantes de medicina brasileiros. Tal estudo reverte-se de importância ao considerar que a população universitária é constituída por jovens oriundos de vários niveis sociais e com educaçảo pré-universitária muito variada. Esses fatos, aliados ao empenho de cada aluno, podem contribuir para a eficácia nos exames cognitivos's.

No cotidiano, há alguns conceitos controversos sobre o desempenho ser melhor entre as alunas, por serem elas mais atentas às obrigaçð̄es discentes e estudarem mais. Por outro lado, considera-se que nas disciplinas de cirurgia os estudantes se destacam mais, por se acreditar que as especialidades cirúrgicas requeiram decisões mais agressivas e menos emocionais, que seriam mais pertinentes ao temperamento masculino. A idade dos universitários é outro assunto polèmico, pois, para alguns, os mais jovens têm um raciocínio mais rápido e conseguem terminar os exames em tempo menor, enquanto os estudantes de idade mais avançada, embora mais lentos, obteriam resultados melhores por causa de sua maturidade. Os esportes trariam um grande beneficio aos alunos, por aliviarem as tensĩes emocionais e os prepararem melhor para competiçðes, mesmo as intelectuais, como os exames discentes. Ainda nesse aspecto, os canhotos sảo tidos como methores esportistas, enquanto os destros e os que apresentam deficiència visual sāo mais dedicados à atividade intelectual. Sendo assim, essas caracteristicas poderiam diferenciar o desempenho dos estudantes.

O objetivo deste trabalho foi conhecer o rendimento de estudantes do curso de medicina na realizaçảo de exame de conhecimento específico e relacionar sua proficiência com as diversas características individuais sobre as quais há conceitos consagrados, porém sem suporte científico. Tendo em vista os conceitos controversos quanto às diferenças de desempenho intelectual entre os sexos, considerou-se adequado avaliar esse parâmetro. Julgou-se também pertinente verificar se alunos que entram na faculdade precocemente teriam, de fato, maior acuidade mental. Caracteristicas individuais - como necessidade de usar óculos, prática de atividade esportiva e lateralidade cerebral - também foram apontadas como fatores potenciais de variaçảo intelectual de acordo com alguns estudos $24.5 .6 .8 .11,84,18,19$.

\section{MÉTODO}

Foram investigados 825 estudantes consecutivos do $6^{2}$ ano da Faculdade de Medicina da UFMG, que voluntariamente se dispuseram a responder o questionário desta pesquisa durante quatro anos. Nesse periodo, houve 16 turmas semestrais de 80 alunos cada, perfazendo um total de 1.280 alunos. A investigação foi realizada tendo por base apenas os questionários completos e respondidos corretamente. Os 203 questionários incompletos ou que levantaram dúvida quanto às respostas foram descartados. Nảo responderam ao questionário 252 alunos, que foram excluidos do presente estudo. Portanto, foram avaliados 825 questionários completos.

Esses alunos foram caracterizados de acordo com a idade, que variou entre 22 e 51 anos, média de $24,6 \pm 6,7$ anos; sexo, $446(54,1 \%)$ homens e $379(45,9 \%)$ mulheres; e lateralidade cerebral, $92,1 \%$ de destros e $7,9 \%$ de canhotos. Os alunos foram distinguidos também de acordo com a prática de esportes: $66,3 \%$ era m sedentários, enquanto $33,7 \%$ praticavam esportes, em média, très vezes por semana, com preferência para os exercicios aeróbicos $(30,7 \%)$, sem diferença entre os sexos. Determinou-se ainda a presença de deficiência visual entre os estudantes: $61,7 \%$ tinham deficiència visual, principalmente miopia $(45,2 \%)$ e astigmatismo $(38,3 \%)$, enquanto os demais referiam visảo perfeita.

$O$ desempenho em conhecimento especifico foi avaliado por meio da prova final do Internato de Cirurgia, constituida por 15 questōes de múltipla escolha, com cinco respostas, sobre toda a matéria teórica ministrada em aula durante os três meses do curso. Foram concedidos $\mathbf{4 5}$ minutos como tempo limite para a realizaçāo dessas provas, sem direito a consulta. Determinou-se a nota de cada aluno e o tempo que ele levou para complelar a prova.

A validade desta pesquisa foi garantida por profissionais da área de estatistica, que orientaram essa parte do trabalho $e$ segundo os quais, mesmo com nú mero reduzido de perguntas, esta pesquisa é pertinente com seus objetivos. A avaliação 
deteve-se nos paràmetros propostos neste trabalho, que buscou esclarecer alguns tópicos controversos sobre o desempenho dos estudantes de medicina. A precisão dos resultados foi conferida pelo elevado número de estudantes investigados. Mediante o teste $t$ para amostras independentes, foram comparados os desempenhos dos estudantes de acordo com as caracteristicas que os identificavam. Considerou-se significativa a diferença maior à correspondente a $p=0,05$.

\section{RESULTADOS}

A Tabela 1 apresenta o desempenho dos estudantes de medicina na prova final do Internato de Cirurgia, de acordo com os parâmetros de sua identificaçāo. A amostra incluiu todos os 825 alunos, e as notas variaram entre a menor obtida no periodo ( 8 pontos) e a maior ( 30 pontos). O menor tempo para concluir a prova foi de 12 minutos, e o maior alcançou o limite dos 45 minutos.

Observa-se que o conceito obtido pelos alunos foi melhor do que o das aluras, com significância para $P=0,051$. Destaca-se ainda que os estudantes com menos de 24 anos obtiveram notas mais elevadas do que os mais velhos $(P=0,006)$. Uma tendência que merece ser apontada é a dos alunos com deficiência visual, que obtiveram notas em geral pouco maiores do que as dos estudantes com visão normal ( $P=0,083$ ). Quanto à lateralidade cerebral, parece que os destros fizeram as provas em menor tempo $(P=0,093)$, sem comprometer sua nota. Contudo, essa comparaçāo é difícil de estabelecer diante do pequeno número de canhotos em relaçāo aos destros.

\section{DISCUSSĀO}

Os questionários foram distribuidos a todos os estudantes do $6^{\circ}$ ano de medicina, e as respostas foram voluntárias. Os quatro anos de pesquisa abrangeram 1.280 alunos, portanto consideramos representativa a amostra de 825 estudantes que responderam o questionário. A espontaneidade da participação neste trabalho confere confiabilidade aos resul$\operatorname{tados}^{3,2,15}$. As características dos estudantes tornam a amostra deste trabalho representativa da Faculdade de Medicina da UFMG e possivelmente dos estudantes de medicina em geral.

Nos últimos anos, cresceu o número de mulheres na Faculdade de Medicina, que passou de menos de $10 \%$, há meio século, para metade e com tendência de superar o número de homens ${ }^{3.8}$. Essa situaçro se deve ao melhor desemperdiu das mulheres, revelado em exames como o vestibular e que se man-

TABELA 1

Avaliação do tempo de realização (máximo de 45 minutos) e da nota obtida em uma prova de cirurgia com 15 questōes de múltipla escolha, no valor de 30 pontos, por 825 estudantes do 60 ano de medicina, de acordo com suas características. Dados apresentados em média e desvio padrāo da média

\begin{tabular}{|c|c|c|c|c|c|}
\hline Parámetro & Grupo & Tempo (min) & $P$ & Nota $/ 30$ & $p$ \\
\hline \multirow[t]{3}{*}{ Sexo } & & & 0,357 & & 0,051 \\
\hline & Masculino $(n=446)$ & $34,5 \pm 8,1$ & & $21,3 \pm 4,2$ & \\
\hline & Feminino $(n=379)$ & $35,0 \pm 7,7$ & & $21,9 \pm 3,9$ & \\
\hline \multirow[t]{3}{*}{ Idade } & & & 0,656 & & 0,006 \\
\hline & $<23 \operatorname{anos}(n=293)$ & $34,6 \pm 7,7$ & & $22,5 \pm 3,9$ & \\
\hline & $>24$ anos $(n=532)$ & $34,8 \pm 7,9$ & & $21,1 \pm 4,0$ & \\
\hline \multirow[t]{3}{*}{ Esporte } & & & 0,137 & & 0,362 \\
\hline & Pratica $(n=278$ ) & $35,3 \pm 7,5$ & & $21,4 \pm 4,1$ & \\
\hline & Nåo pratica $(n=547)$ & $34,4 \pm 8,0$ & & $21,7 \pm 4,0$ & \\
\hline \multirow[t]{3}{*}{ Deficiência visual } & & & 0,885 & & 0,083 \\
\hline & Presente $(n=509)$ & $34,1 \pm 8,2$ & & $21,7 \pm 4,0$ & \\
\hline & Ausente $(n=316)$ & $34,0 \pm 7,4$ & & $22,3 \pm 3,8$ & \\
\hline \multirow[t]{3}{*}{ Lateralidade cerebral } & & & 0,093 & & 0,109 \\
\hline & $\operatorname{Destro}(n=760)$ & $34,3 \pm 8,0$ & & $21,9 \pm 3,3$ & \\
\hline & Canhoto $(n=65)$ & $36,3 \pm 6,5$ & & $22,1 \pm 4,1$ & \\
\hline
\end{tabular}

$P$-Refere-se ao testet para amostras independentes, correspondente a cada pardmetro avaliado. Os valores se referem ds comparacöes cas medias apresentadas nas duas subsequientes anteriores.

Menor nota: 8 pontos; maior nota: 30 pontos.

Menor tempo: 12 minutos; maior tempo: 45 minutos. 
tém durante o curso superior, mas é pertinente supor que não haja diferença na capacidade intelectual entre os sexos. As methores notas foram obtidas pelos homens, mas é fundamental apontar que a prova era de cirurgia, disciplina que provavelmente desperta menor interesse em estudantes do sexo feminino. Essa hipótese pode ser subsidiada pelo maior número dehomens que se inscrevem para Residência em especialidades cirúrgicas ${ }^{16}$.

A elevada significância que caracterizou o melhor desempenho dos alunos mais jovens pode ser atribuida a caracteristicas individuais. Esses estudantes provavelmente foram também os que se destacaram mais na vida estudantil prévia, sem reprovação nos cursos elementai, secundário e vestibular, bem como nas outras disciplinas do curso de medicina. Muitos fatores podem ter contribuido para que esses alunos obtivessem melhor nota, como nivel intelectual, seriedade e dedicaçāo maior aos estudos, além de gosto pelo aprendizado. Esses atributus predispõem à obtençâo de resultados favoráveis mais precocemente. Por outro lado, em termos relativos, as médias das notas dos alunos não diferiram muito entre si, situandose numa faixa ao redor de $70 \%$.

O objetivo de incluir a prática de exercícios físicos nesta pesquisa deveu-se ao fato de os esportistas serem competitivos e mais objetivos em suas metas. Sendo assim, acreditávamos que, nas provas de cirurgia, disciplina também prática e com caracteristicas de desafio, os alunos que praticam esportes teriam melhor desempenho. Pensávamos também que a atividade física contribuisse para uma velocidade de pensamento maior, com conseqüente redução no tempo de realização da prova. Entretanto, na comparação do desempenho relacionado a exercício físico, não houve diferença entre quem pratica esportes e ns que têm uma vida sedentária, tanto na nota quanto no tempo de prova.

Embora não tenha sido o objetivo principal desta investigação, é importante ressaltar a reduzida prática esportiva dos estudantes de medicina de ambos os sexos na época da vida de maior capacidade física. Essa tendência ao sedentarismo eleva bastante o risco futuro de doenças cardiovasculares, principalmente se for considerada a falta de cuidados com a dieta e o tabagismo, muito freqüente entre os médicos. Além dos perigos para si, com essa conduta negligente, o médico pode, como modelo social, ser um exemplo negativo para sua familia e as pessoas de seu relacionamento, incluindo os pacientes ${ }^{17,18.19}$. Cabe, portanto, o alerta à classe médica, por meio de suas agremiaçōes, para esse problema, que de forma alguma deve ser considerado secundário.

A deficiência visual também se relacionou com a tendência a melhores notas na prova. Mesmo não sendo significati- va a diferença, os alunos com deficiência visual tiveram um desempenho melhor. Uma das hipóteses para explicar essa observação pode ser a caracteristica de maior introversão de quem é limitado pelo uso de óculos e sua maior facilidadeem se concentrar no estudo. Tal constatação não deve ser considerada uma regra, mas apenas uma propensão. Por outro lado, cabe salientar que a prevalència de alunos com deficiência visual na Faculdade de Medicina foi muito superior à encontrada na população geral de mesma faixa etária e que gira em torno de $14 \%$. Essa situação precisa ser mais bem estudada, para tentar esclarecer os motivos que se relacionam com a deficiência visual entre os universitários.

$O$ reduzido número de canhotos encontrado entre os estudantes não permite uma análise mais profunda dos resultados. A tendência de os destros fazerem a prova em um tempo menor pode ser um fato isolado, sem relação com a lateralidade cerebral.

Os resultados desta peequisa não devem ser considerados parametros definitivos para uma orientaçāo educacional, tendo em vista a ampla variedade de parâmetros que envolvem a resolução de um exame. Podem existir outros fatores de impacto eventualmente maior, que, se ben analisados, se mostrem mais significativos na influência dos alunos diante de um exame. Entretanto, o objetivo maior deste trabalho foi verificar a veracidade científica do papel de algumas características individuais no desempenho de estudantes de medicina no exame final do Internato de Cirurgia. Constatamos que, apesar de consagrado no cotidiano, o valor desses atributos $e ́$, de fato, pequeno e sua influência não é marcante na atuação intelectual desses alunos.

Caracteristicas individuais contribuem para os diferentes desempenhos que os estudantes de medicina apresentam nas provas. Os resultados deste trabalho levantam múltiplas questỏes, principalmente voltadas aos estudantes mais jovens, à grande prevalência de deficiência visual e ao reduzido interesse dos estudantes por atividade fisica, que precisam ser mais bem investigadas em pesquisas futuras.

\section{REFERÊNCIAS BIBLIOGRÁFICAS}

1. Petroianu A. Relação entre a escolha da especialidade médica e a preferência por matérias do curso secundário. J Bras Méd 1993; 65: 161-166.

2. Searle J, McHarg J. Selection for medical school. Med Educ 2003; 37: 458-463.

3. Petroianu A. A pesquisa em Medicina. Medicina 1992; 25 : 327-329.

4. Reed VA, Jernstedt C, Boudreau D, Dollase $\mathrm{RH}$, Nierenberg DW, Shelton W, Jacoby L. Comprehensive measurement 
of profiles of student characteristics across medical schools. Acad Med 1999; 74: S96-S98.

5. Hojat M, Gonnella JS, Edmann JB, Veloski JJ. The fate of medical students with different levels of knowledge. Adv Health Sci Educ 1997; 1: 179-196.

6. Mavis B. Self-eff.cacy and OSCE performance among second year medical students. Adv Health Sci Educ 2001;6: 93-102.

7. Pereira WJF, Petroianu A, Brito AO, Oliveira CHC, Silva IFAFR, Canela GGC, Gontijo GHS. Avaliaçāo do uso de drogas por estudantes de Medicina. Rev Med Minas Gerais 2000; 10: 8-12.

8. Pereira WJF, Petroianu A. Interesse de estudantes de Medicina por pesquisacientifica. Rev Bras Educ Méd 2000; 24: 9-13.

9. Chang DF, Sue $S$. The effect of race and problem type on teachers' assessments of student behavior. J Consult Clin Psychol 2003; 71: 235-242.

10. DaRosa DA, Niehaus AH, Markwell SJ. Assessment of a surgery clerkship's performance evaluation system. Am J Surg 2000; 179: 145-149.

13. Niemi PM. Medical students' professional identity. Med Educ 1997; 31: 108-415.

12. Collins JP, Gamble GD. A multi-format interdisciplinary final examination. Med Educ 1996; 30: 259-265.

13. Curran V, Christopher J, Lemire F, Collins A, Barrett B. Application of a responsive evaluation approach in a medical education. Med Educ 2003; 37: 256-266.
14. Fitzgerald TT, Gruppen LD, White CB. The influence of task formats on the accuracy of medical students' selfassessments. Acad Med 2000; 75-737-7.11.

15. Petroianu A. Ética moral e deontologia médicas. Rio de Janeiro: Guanabara Koogan; 2000.

16. Vieira MI, Petroianu A. Distribuiçāo dos médicos no Estado de Minas Gerais. An Acad Med 1996; 156: 38-41.

17. Moses J, Steptoe A, Mthews A, Edwards S. The effects of exercise training on mental well-being in the normal population. J Psychosom Res 1999; 33: 47-61.

18. Zervas $Y$, Danis A, Klissouras V. Influerce of physical exertion on mental performance with reference to training. Percept Motor Skills 1991; 72: 1215-1221.

19. Christensen $H$, Mackinnon $A$. The association between mental social and physical activity and cognitive performance in young and old subjects. Age Ageing 1993; 22: 175-182.

\section{Endereço para correspondência}

Andy Petroianu

Avenida Afonso Pena, 1626 - apte 1.901

30130-005 - Belo Horizonte - MG

Tel. / fax (31) 3274-7744

E-mail: petroian@medicina.ufmg.br 